\title{
Velocity of galaxies with different luminosity
}

\author{
Y. P. Jing ${ }^{1}$ and G. Börner ${ }^{2}$ \\ ${ }^{1}$ Shanghai Astronomical Observatory, the Partner Group of MPI für Astrophysik, \\ Nandan Road 80, Shanghai 200030, China email: ypjing@shao.ac.cn \\ ${ }^{2}$ Max-Planck-Institut für Astrophysik, Karl-Schwarzschild-Strasse 1, \\ 85748 Garching, Germany email: grb@mpa-garching.mpg.de
}

\begin{abstract}
We present the first determination of the pairwise velocity dispersion of galaxies at different luminosity with the final release of the Two-Degree Field Galaxy Redshift Survey (2dFGRS). Our result surprisingly shows that the random velocities of the faint galaxies are very high, around $700 \mathrm{~km} \mathrm{~s}^{-1}$, reaching similar values as the brightest galaxies. At intermediate luminosities slightly brighter than the characteristic luminosity $M_{\star}$, the velocities exhibit a well defined steep minimum near $400 \mathrm{~km} \mathrm{~s}^{-1}$. The result challenges the current halo model of galaxies of Yang et al. that was obtained by matching the clustering and luminosity function of 2dFGRS, and can be an important constraint in general on theories of galaxy formation, e.g., the semi-analytical model. Combining the observed luminosity dependence of clustering, our result implies that quite a fraction of faint galaxies are in massive halos of galaxy clusters as the brightest ones, but most of the $M_{\star}$ galaxies are in galactic halos.
\end{abstract}

\section{Introduction}

The clustering of galaxies in the Universe is characterized by their spatial positions, and their peculiar velocities which lead to deviations of their motion from the pure Hubble flow. The big redshift surveys assembled in recent years by the diligent work of many astronomers give angular positions and redshifts for large numbers of galaxies. A rough 3D map can be obtained by placing the galaxies at distances along the line of sight derived via Hubble's law from their redshifts. The peculiar velocity, however, also contributes to the redshift, and this leads to a misplacement of the galaxy away from its true location. The local gravitational field is the cause of the peculiar motion and thus the redshift distortion in the galaxy maps can give information on the underlying matter distribution.

The amplitude of the distortions can be estimated from the pair distribution of galaxies. For pairs of galaxies at distances much larger than their separation, one can use a planeparallel approximation, and write for the power spectrum in redshift space,

$$
P^{S}(k, \mu)=P(k) \frac{\left(1+\beta \mu^{2}\right)^{2}}{1+\frac{1}{2}\left[\sigma_{v}(k) k \mu\right]^{2}} .
$$

Here $\mu$ is the cosine of the angle between the wave vector and the line of sight. The linear redshift distortion parameter $\beta$ is related to the linear growth factor $f\left(\Omega_{M}\right) \simeq \Omega^{0.6}\left(\Omega_{M}\right.$ is the matter density), and the normalization $\sigma_{8}$ of the density fluctuation spectrum via $\beta=\Omega_{M}^{0.6} \sigma_{8} . P(k)$ is the power spectrum in real space. The pairwise velocity dispersion (PVD) $\sigma_{v}(k)$ describes the virial motion of galaxies in dense systems, i.e. the Fingerof-God effect. As shown by Jing \& Börner (2001), the redshift power spectrum of dark matter and its biased tracers can be accurately described by Eq.(1.1). Applying this model to an observation of $P^{S}(k, \mu)$ may therefore yield a determination of the three quantities $P(k), \beta$, and $\sigma_{v}(k)$, all of which are useful observables for testing galaxy formation models. 
Here we use the final release of the 2dFGRS (http://www.mso.anu.edu.au/2dFGRS) to measure $P^{S}(k, \mu)$ and the PVD $\sigma_{v}(k)$ of galaxies. Since we are interested in the PVD at small scale, we fix $\beta$ at a reasonable value of 0.45 (Peacock et al. 2001). The $2 \mathrm{dF}$ catalog has already been analyzed statistically with respect to the PVD (Hawkins et al 2003), but here we use a novel method to estimate the PVD. Furthermore, we take advantage of the large number of galaxies in the $2 \mathrm{dF}$ catalog to bin the galaxies in different luminosity intervals, and to make the first study of the luminosity dependence of the PVD. This was not possible up to now, and we shall see that remarkable tests of the galaxy formation models become possible with the luminosity dependence of the PVD. The luminosity dependence of the clustering (the two-point correlation function) of galaxies in the 2dFGRS has been investigated (Norberg et al. 2002a), but not of the PVD.

\section{Statistical analysis}

In order to study the luminosity dependence of the PVD, we divide the galaxies into 11 subsamples according to their absolute luminosity. The subsamples are successively brightened by 0.5 magnitude from the faintest sample $M_{b}=-17.0+5 \log h$ to $M_{b}=$ $-22.0+5 \log h$, with successive subsamples overlapping by 0.5 magnitude. Here $h$ is the Hubble constant in units of $100 \mathrm{~km} \mathrm{~s}^{-1} \mathrm{Mpc}^{-1}$. For computing the absolute magnitude, we have used the k-correction and luminosity evolution model of Norberg $(2002 \mathrm{~b} ; \mathrm{k}+\mathrm{e}$ model); i.e., the absolute magnitude is in the rest frame $b_{j}$ band at $z=0$. We assume a cosmological model with the density parameter $\Omega_{0}=0.3$ and the cosmological constant $\lambda_{0}=0.7$ throughout this paper.

We measure the redshift two-point correlation functions $\xi_{z}(\mathbf{s})$ following the method of Jing, Mo, \& Börner(1998). The random samples for the clustering analysis are generated in the same way as described in Jing \& Börner (2004a). Each random sample for a northern or southern luminosity subsample contains 100,000 random points. We convert the $\xi_{z}(\mathbf{s})$ to $P^{S}(k, \mu)$ by the Fourier transformation. More details about our statistical method can be found in our journal paper (Jing \& Börner 2004b).

\section{Results}

In Fig. 1 the basic measurement of the power spectrum in redshift space $P(k, \mu)$ is shown. The four panels in this figure correspond to four different luminosity intervals from faint to bright galaxies. The values of $\mathrm{k}$ range from $0.16 \mathrm{hMpc}^{-1}$ at the top to $2.5 \mathrm{hMpc}^{-1}$ at the bottom with an interval $\Delta \log _{10} k=0.2$. The south and north samples agree quite well with the full survey indicating that cosmic variance is not a major problem. The power spectrum for the larger k-values decreases quite strongly with $\mu$, more than a factor of 10 between $\mu=0$ and $\mu=1$. The solid lines are the best fits obtained by applying equation (1.1) to the data of the whole survey, showing that the model (1.1) can accurately describe the observed redshift power spectrum of galaxies.

The luminosity dependence of the PVD is shown most clearly in Fig. 2, where we have plotted $\sigma_{v}$ at $k=1 \mathrm{hMpc}^{-1}$ for the 11 overlapping samples. The surprising result is a strong dependence on luminosity with the bright and the faint galaxies reaching high values of $\simeq 700 \mathrm{~km} \mathrm{~s}^{-1}$ or more, and a well defined minimum of $\simeq 400 \mathrm{~km} \mathrm{~s}^{-1}$ for the galaxies of magnitude $M_{b}-5 \log _{10} h=-20.5$. The bright and the faint galaxies apparently have high random motions, as expected for objects in massive halos or in clusters. The $M_{\star}$ like galaxies are rather moderate in their PVD, and probably reside in galaxy size halos. 


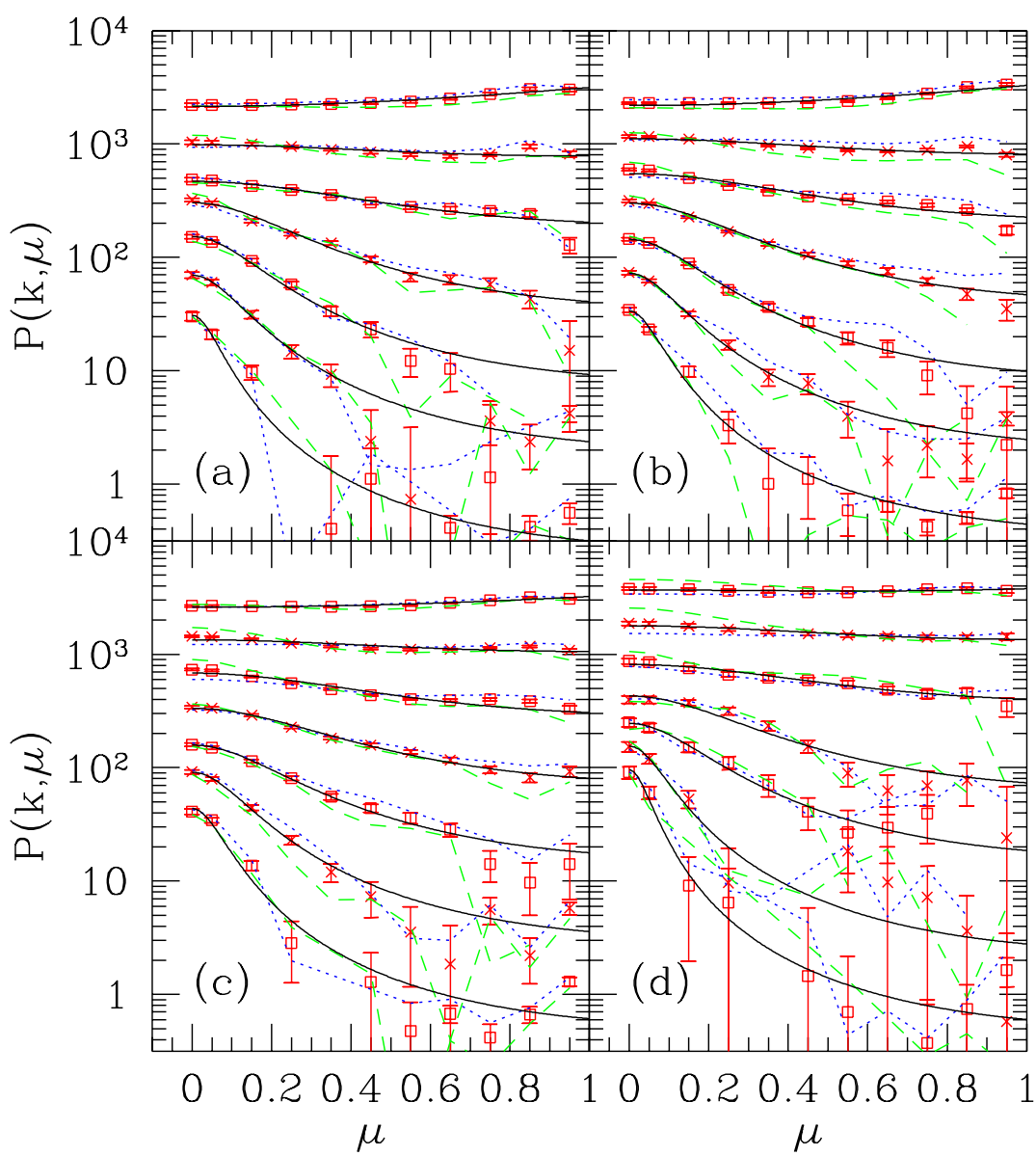

Figure 1. The redshift space power spectrum $P(k, \mu)$ measured in 2dFGRS. The symbols are for the whole survey, the dotted lines for the south subsample, and the dashed lines for the north subsample. The errors are plotted only for the whole survey, estimated with the bootstrap method. The smooth solid lines are the best fits of Eq.(1.1) to data of the whole sample. (a) for $-17.5<M_{b}-5 \log h<-18.5$; (b) for $-18.5<M_{b}-5 \log h<-19.5$; (c) for $-19.5<M_{b}-5 \log h<-20.5$; (d) for $-20.5<M_{b}-5 \log h<-21.5$. The $k$ values in each panel are from $0.16 \mathrm{hMpc}^{-1}$ (top) to $2.5 \mathrm{hMpc}^{-1}$ (bottom).

The thick solid line represents the prediction based on the up-to-date halo model of Yang et al. (2003). For the halo model, we adopt the cosmological model that is a currently popular flat low-density model with the density parameter $\Omega_{0}=0.3$ and the cosmological constant $\lambda_{0}=0.7(\mathrm{LCDM})$. The shape parameter $\Gamma=\Omega_{0} h$ and the amplitude $\sigma_{8}$ of the linear density power spectrum are 0.2 and 0.9 respectively. We use two sets of simulations, with boxsizes $L=100 h^{-1} \mathrm{Mpc}$ and $L=300 h^{-1} \mathrm{Mpc}$, that were generated with our vectorized-parallel $\mathrm{P}^{3} \mathrm{M}$ code (Jing \& Suto 2002) for this model. Both simulations use $512^{3}$ particles, so the particle mass $m_{p}$ is $6.2 \times 10^{8} M_{\odot}$ and $1.7 \times 10^{10} M_{\odot}$ respectively in these two cases. We populate the halos with galaxies in a similar way to that of Yang et al. (2004), but we used our own code and adopted the model parameters of Model M1 in Yang et al. (2003). The halo model, which matched nicely the luminosity function and the luminosity dependence of clustering of galaxies in 2dFGRS (confirmed by our analysis), clearly does not match our observation of the PVD. The failure of the 


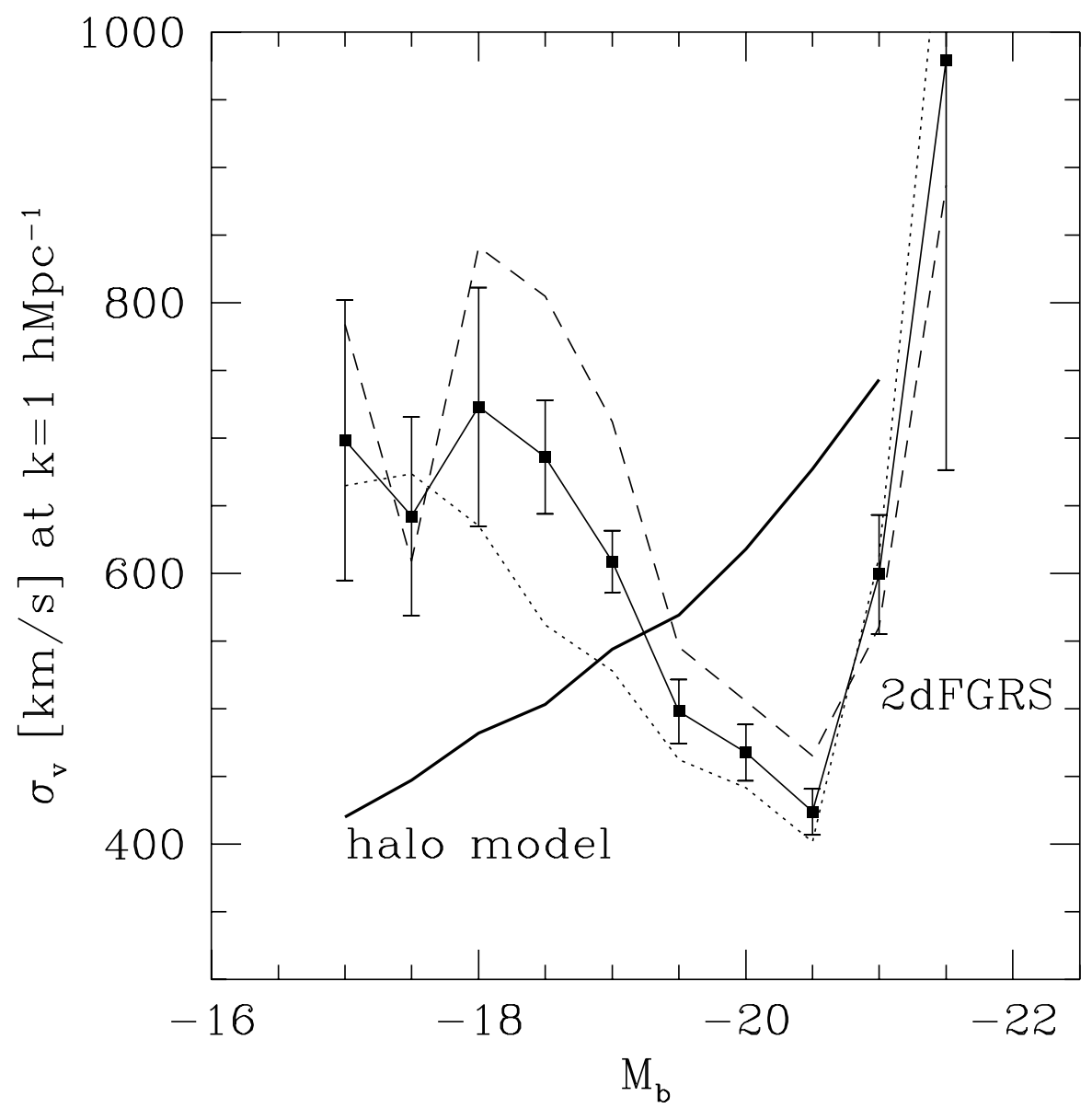

Figure 2. The pairwise velocity dispersion measured at $k=1 \mathrm{hMpc}^{-1}$ in the $2 \mathrm{dFGRS}$ (symbols for the whole sample, dotted line for the south, and dashed line for the north), compared with the prediction based on the halo model (thick solid line). The error bars of the observed results are given by the mock samples.

halo model indicates that the prescription of how to populate halos must be adapted better.

\section{Discussion}

The analysis of the velocity fields of the galaxies in the 2dFGRS has led to a surprising discovery: the random velocities of the faint galaxies are very high, around $700 \mathrm{~km} \mathrm{~s}^{-1}$, reaching similar values as the bright galaxies. At intermediate luminosities the velocities exhibit a well defined steep minimum near $400 \mathrm{~km} \mathrm{~s}^{-1}$.

It seems that the galaxies in different luminosity intervals appear as different populations in their own right, defined by objective statistics. A look at figure 2 shows convincingly that this is actually the case. For this figure we have sorted the galaxies in 11 luminosity bins, each one magnitude wide, from magnitude -16.5 to -22 and plotted the value of $\sigma_{v}$ at a wave number of $k \simeq 1 \mathrm{hMpc}^{-1}$. Such a finely resolved binning of galaxies in samples of different luminosities is possible for the 2dFGRS, because it is big enough to contain sufficiently many galaxies in each luminosity class. 
The PVD is an indicator of the depth of the local gravitational potential. Therefore we find the interesting result that the bright and the faint galaxies move in the strongest gravitational field. They are in clusters, while the galaxies around magnitude -20 , the $M_{*}$ galaxies in the Schechter luminosity function, populate the field.

The bimodal nature of the correlation between the PVD and luminosity may be used as a stringent test of galaxy formation models. We have investigated the halo occupation model (Yang et al. 2003) which has been optimally fitted to reproduce the luminosity function, and the two-point correlation function of the 2dFGRS. If we adapt this model to the PVD value of the $M_{*}$ galaxies, we see that it cannot give the high values found for the fainter galaxies. The PVD values of the model actually run opposite to the data and the model assigns smaller values to the fainter galaxies. This must mean that the assignment of galaxies to the dark matter halos must be done in a more intricate way as up to now. The number of faint galaxies in clusters must be increased substantially to at least recover the high PVD found for them. Also, the low value of $400 \mathrm{~km} \mathrm{~s}^{-1}$ found for the galaxies with magnitude -20.5 must mean that these galaxies reside in dark matter halos of galactic size. The halo population model must be fitted with a much more complex scheme of assigning galaxies to halos, if the results shown in figure 2 are to be reproduced.

Another way, widely used, to connect dark matter to galaxies is the semi-analytic modeling, where the dark matter distributions obtained from N-body simulations are supplemented with some of the physical processes important in galaxy formation using semi-analytic techniques. A test of the PVD vs luminosity for this type of models will be the aim of a subsequent paper.

\section{Acknowledgements}

JYP would like to thank the Max-Planck Institute für Astrophysik for its warm hospitality, IAU for its partial travel support, and the SOC for adjusting the talk time. The work is supported in part by NKBRSF (G19990754), by NSFC (No.10125314), and by the CAS-MPG exchange program.

\section{References}

Hawkins, E., et al. 2003, MNRAS, 346, 78

Jing, Y. P. \& Börner, G. 2001, ApJ, 547, 545

Jing, Y. P. \& Börner, G. 2004a, ApJ (in press) astro-ph/0311585

Jing, Y. P. \& Börner, G. 2004b, in preparation

Jing, Y. P., Mo, H. J., \& Börner, G. 1998, ApJ, 494, 1

Jing, Y. P. \& Suto, Y. 2002, ApJ, 574, 538

Norberg, P. et al. 2002a, MNRAS, 332, 827

Norberg, P. et al. 2002b, MNRAS, 336, 907

Yang, X. et al. 2004, MNRAS (in press) astro-ph/0303524

Yang, X., Mo, H. J., \& van den Bosch, F. C. 2003, MNRAS, 339, 1057

Peacock, J. A., et al. 2001, Nature, 410, 169 